\title{
Prenatal ultrasonographic diagnosis of urethral atresia
}

\author{
N B Thapa ${ }^{1}$, Y Dwa ${ }^{2}$, S Gurung ${ }^{2}$, R Baral $^{3}$ \\ ${ }^{1}$ Department of Radiology; ${ }^{2}$ Department of Obstetrics and gynecology; ${ }^{3}$ Department of Pathology, KIST Medical College, \\ Lalitpur, Nepal.
}

Correspondence to: Narayan Bikram Thapa, Department of Radiology, Lecturer, KIST Medical College, Imadol.

Email-nadudocradio@yahoo.com

\begin{abstract}
Urethral atresia is one of the rare congenital anomaly of urogenital tract. In this case report, uretheral atresia diagnosed antenatally by ultrasonography (USG) at 24-25 weeks of gestation is presented. USG findings were confirmed by postmortem examination of the fetus.
\end{abstract}

Key words: Lower urinary tract obstruction, posterior urethral valves, ultrasonography, urethral atresia,

\section{Introduction}

The development of the genitourinary system is complex. Numerous anomalies of the urethra exist, either as isolated anomaly or in combination with other disorders. Various anomalies resulting in lower urinary tract obstruction (LUTO) has been documented in the literature isolated like posterior urethral valves, urethral stricture, urethral atresia, urethral membranes, urethral polyps, mullerian duct remnants like enlarged prostatic utricle and mullerian duct cyst, cowper's syringocele, anterior urethral valves and diverticula, megalourethra, urethral duplication and urethroperineal fistula and in combination with other disorders like prune-belly syndrome and anorectal malformations. Among these the posterior urethral valves is the commonest one. ${ }^{1}$

Urethral atresia is one of the rare congenital abnormality often found in association with other genitourinary anomaly but it is also found as an isolated anomaly. ${ }^{2}$ In literature, the urethral atresia is not always clearly defined, whether an absence or a partial absence stays unclear. ${ }^{3}$ It occurs more commonly in male than in female and in male; it is often difficult to differentiate between uretheral atresia and posterior urethral valves.

Routine antenatal USG can diagnose uretheral obstruction, severity of obstruction and other associated congenital anomaly of urogenital tract. In this case report we highlight the ultrasonographic findings of urethral atresia on antenatal scanning along with photographs of aborted fetus.

\section{Case}

A 24 year old primigravida was referred at 26 weeks of gestation for antenatal ultrasonographic scanning. There was no history of intake of any drugs or radiation exposure.

Ultrasound scan revealed a live single fetus in cephalic presentation. The gestational age was estimated to be about 24-25 weeks according to biparietal diameter and fetal long bone measurements. On routine survey of the fetus, the fetal heart motion and fetal movements were normal. Examination of the fetal abdomen showed a normal liver, stomach and intestines. Fetal kidneys showed evidence of dilatation of collecting system in both sides (RT $>$ LT). Both the kidneys were grossly enlarged and there is thin rim of cortex in right side and echogenic parenchyma in left side suggestive of renal dysplasia. On tracing the ureters, they were found to be dilated on both sides. The fetal bladder was distended (Fig. 1). There was typical 'Keyhole sign' due to grossly distended urinary bladder and dilatation of proximal urethra (Fig. 2). This was observed even after examination for half an hour. The fetus was identified to be male by visualization of the scrotal shadow and penis. The placenta was normal. Lungs were normal and no other fetal anomalies were detected. A provisional diagnosis of obstruction at urethral level causing bilateral hydronephrosis, hydroureter and megacystis was made. Either posterior uretheral valve or uretheral atresia is considered as a possible underlying anomaly.

The patient was informed about poor prognosis of the fetus 
and after counseling; she elected to terminate the pregnancy. Male fetus along with complete placenta and membranes were expelled.

Taking the consent from the mother, detail postmortem examination of the fetus was carried out to identify underlying anatomical abnormality. Distal urethra was atretic (Fig. 3) with dilated proximal urethra, grossly distended urinary bladder, along with bilateral hydroureter and enlarged hydronephrotic kidneys (Fig. 3). The left kidney was dysplastic. No other congenital abnormalities noted. Umbilical cord was normal and there were two arteries and one vein.

After delivery mother was informed that there was no known recurrence risk. She was counseled to report to hospital for regular screening in her future pregnancies.

\section{Discussion}

There are many causes of LUTO in the fetus. Many of these conditions are difficult to differentiate during an antenatal USG. Whatever is the cause for LUTO, it is always necessary to determine whether underlying anomaly will be compatible with life or not which depends upon backpressure changes in upper urinary tract caused by LUTO and other associated congenital anomaly. If the obstruction is intermittent or partial, only back pressure changes can be seen in bladder, ureters and kidneys while if severe and long standing cases, progressive renal fibrosis and dysplasia develop that leads to severe oligohydramnios, pulmonary hypoplasia and compression deformities. ${ }^{4}$ Uretheral atresia associated with renal dysplasia and/or pulmonary hypoplasia is almost always lethal. ${ }^{5}$ In our case also there was associated renal dysplasia which is lethal condition. The most important after diagnosis of LUTO in fetus is to find out whether it is lethal or not. The most common cause LUTO in fetus is posterior urethral valves (PUV) so in any cases of LUTO, posterior urethral valve is to be ruled out. In our case also, as the fetus was male and being most common lower urinary tract anomaly, posterior urethral valve was considered as a cause for urethral obstruction but severe oligohydramnios is less common with posterior urethral valve so urethral atresia was also kept as a possible underlying cause for lower urinary tract obstruction. The prognosis of lower urinary tract obstruction will depends upon the time of diagnosis and available intervention, associated other congential anomalies and post delivery available surgical facility and its outcome. In fetus with LUTO detected in first trimester or early in second trimester and without other lethal congenital malformation, urethral decompression by intrauterine placement of vesicoamniotic shunt can be tried however small number of studies shows $30 \%$ survival rate. ${ }^{6}$

\section{Conclusions}

Numerous anomalies of urethra can results in lower urinary tract obstruction. Severity of obstruction and associated congenital abnormalities are important factor for the prognosis of the fetus.

\section{References}

1. Terry L Levin,Bokyung Han,Brent P. Little. Congenital anomalies of the male urethra. Pediatr Radiol 2007;37:851-862.

2. Twinning P Urinary tract abnormalies. Textbook of fetal anomalies. 2000;02:300-301.

3. Mogg RA. Congenital anomalies of the urethra. Br J Urol.1968:40:638-9.

4. Glazer GM, Filly RA, Callen PW. The varied sonographic appearance of urinary tract in the fetus and newborn with urethral obstruction. Radiology 1982;144:563-568.

5. Bierkens AF, Feitz WFJ,Nijhuis JG et al. Early urethral obstruction sequence: a lethal entity? Fetal Diagn Ther 1996;11:137-145.

6. Freedman AL, Bukowski TP, smith CA et all. Fetal therapy for obstruction. Urol 1996; 156: 72. 